\title{
Antibiotic resistance and antibiotic sensitivity based treatment in Helicobacter pylori infection: advantages and outcome
}

\author{
M E Street, P Caruana, C Caffarelli, W Magliani, M Manfredi, F Fornaroli, \\ G L de'Angelis
}

\begin{abstract}
Aims-To compare two strategies for the eradication of Helicobacter pylori infection.

Methods-Groups 1 and 2 each consisted of 75 consecutive patients. Patients in group 1 were treated with two antibiotics based on antibiotic susceptibility testing; those in group 2 received amoxycillin and clarithromycin for eight days, together with either ranitidine or omeprazole. Eradication rate was assessed in both groups six months after treatment.

Results-In group $1, H$ pylori grew in culture in $63 / 75$ cases. Susceptibility testing showed that $35 / 63$ isolates were resistant to metronidazole, $10 / 63$ to clarithromycin, $2 / 63$ to ampicillin, 1/63 to tetracycline, and $5 / 63$ to both clarithromycin and metronidazole. In group 1 the infection was eradicated in $96 \%$ of the initial 75 subjects, and in $98 \%$ of the subjects treated according to the antibiotic assay (62/63). As two patients were lost at follow up the overall eradication rate was $99 \%$. In group 2, eradication was achieved in $61 / 75$ subjects $(81 \%)$. This was significantly lower than the percentage of eradication observed in group 1 ( $81 \%$ versus $99 \%)$.

Conclusions-Antibiotic susceptibility tests are useful in childhood as a very high percentage of subjects are cured. This approach is costly, but selective antibiotic treatment contributes to limit further development of antibiotic resistance, and money is saved in terms of reinvestigation and further repeated treatments. (Arch Dis Child 2001;84:419-422)
\end{abstract}

Keywords: H pylori; antibiotic resistance; antibiotic susceptibility assay; eradication

Helicobacter pylori is an important bacterial pathogen. It is widely believed that this common infection should be eradicated because it has a key role in several gastric and duodenal diseases, including peptic ulceration and gastric cancer. ${ }^{12}$

Combined antibiotic therapy is routinely used, but antibiotic resistance is an increasing problem, and $H$ pylori may be resistant to commonly used antibiotics such as metronidazole, clarithromycin, and occasionally amoxycillin..$^{3-13}$ In $1996-97$, the reported eradication rate of $H$ pylori in children treated with triple therapy (antisecretory agent and two antibiotics) for at least two weeks ranged from $70 \%$ to $80 \%$. $^{14}$ The standard criteria for treatment of adults are those published by the 1996 Digestive Health Initiative International Update Conference ${ }^{15}$ and by the 1997 Maastricht Consensus Report. ${ }^{16}$ However, these are not entirely applicable to children.

The aim of the present study was to assess the efficacy of combined antibiotic treatment based on the results of antibiotic susceptibility testing using endoscopic gastric biopsy specimens from children with $H$ pylori infection, versus standard blind triple therapy.

\section{Materials and methods}

A total of 150 children with $H$ pylori infection were enrolled consecutively in the study, over a period of two years (January 1997 to December 1998).

The first 75 subjects (group 2) were treated with a standard antibiotic therapy regimen because antibiotic susceptibility testing was not available at that time. The following 75 (group 1) were treated if possible with an antibiotic regimen based on the results of antibiotic susceptibility testing. All patients had symptoms and/or positive serology and/or positive urea breath test at presentation.

Group 1 consisted of 75 patients (age range 2-17 years, 45 males, 30 females). Group 2 consisted of 75 subjects (age range 3-18 years, 49 males, 26 females). The diagnosis of $H$ pylori infection was based on the endoscopic appearance of the gastric mucosa, gastric histology, culture of endoscopic biopsy specimens, and rapid urease test. The histology was classified according to the updated Sidney Score System. ${ }^{17}$

Endoscopic biopsy specimens were taken in all subjects for: (1) histology (two from the gastric antrum, and one from the gastric fundus); and (2) rapid urease test (one from the antrum) (CP test, Yamanouchi Pharma $\mathrm{SpA}$ ). From patients in group 1, two further biopsy specimens were taken from the antrum for microbiological culture. The biopsy specimens for histology were fixed in formalin, embedded in paraffin, sectioned, and stained with haematoxylin-eosin. The biopsy specimens for the bacterial culture were immediately placed in an appropriate transport medium (Portagerm-Pylori, bioMérieux, France), then homogenised and cultured on both selective (Pylori agar, bioMérieux) and non-selective ( $10 \%$ horse blood agar, Kima, Italy) media. After seven days of incubation at 
Table 1 Features at diagnosis of the patients in groups 1 and 2

\begin{tabular}{llll}
\hline & Group 1 $(n=75)$ & Group 2 $(n=75)$ & p value \\
\hline Symptoms & & & \\
$\quad$ Epigastric pain & $29(39 \%)$ & $39(52 \%)$ & NS \\
Recurrent abdominal pain (RAP) & $41(55 \%)$ & $49(65 \%)$ & NS \\
RAP with vomiting & $28(37 \%)$ & $18(24 \%)$ & NS \\
Gastric pyrosis & $9(12 \%)$ & $7(16 \%)$ & NS \\
Anorexia & $6(8 \%)$ & $5(7 \%)$ & NS \\
Epigastric bloating & $9(12 \%)$ & $4(5 \%)$ & NS \\
Bleeding of the upper GI tract & $2(3 \%)$ & $1(1 \%)$ & NS \\
Melaena & $1(1 \%)$ & $1(1 \%)$ & NS \\
Histology & $63(84 \%)$ & $60(80 \%)$ & NS \\
Mild gastritis & $6(8 \%)$ & $10(13 \%)$ & NS \\
Moderate gastritis & $6(8 \%)$ & $5(7 \%)$ & NS \\
Pronounced gastritis & $70(93 \%)$ & $75(100 \%)$ & NS \\
H pylori positive & $70(93 \%)$ & $64(85 \%)$ & NS \\
Positive urease tests & $63(84 \%)$ & Not done & \\
Positive cultures & $58(77 \%)$ & $53(71 \%)$ & NS \\
Endoscopic appearance of the mucosa & $3(4 \%)$ & $2(3 \%)$ & NS \\
Antral nodularity and/or hyperaemia & Duodenal ulcer & & \\
\hline
\end{tabular}

Table 2 Antibiotic resistance to $H$ pylori, based on the E test performed on the positive cultures (63/75) from the endoscopic biopsy specimens from patients in group 1

\begin{tabular}{lll}
\hline Antibiotic & $\begin{array}{l}\text { MIC interpretative values }(\mu g / m l) \\
R\end{array}$ & $\begin{array}{l}\text { Resistance } \\
n(\%)\end{array}$ \\
\hline $\begin{array}{l}\text { Metronidazole } \\
\text { Clarithromycin }\end{array}$ & $\geqslant 16$ & $35(56 \%)$ \\
Ampicillin & $\geqslant 4$ & $10(16 \%)$ \\
Tetracyclines & $\geqslant 16$ & $2(3 \%)$ \\
Metronidazole + clarithromycin & & $1(2 \%)$ \\
Total & & $5(8 \%)$ \\
\hline
\end{tabular}

The isolates with resistance to both metronidazole and clarithromycin are in addition to those with resistance to metronidazole or clarithromycin alone.

Table 3 H pylori eradication rate in groups 1 and 2

\begin{tabular}{llll}
\hline & $\begin{array}{l}\text { Group 1 } \\
(n=75)\end{array}$ & $\begin{array}{l}\text { Group 2 } \\
(n=75)\end{array}$ & $\begin{array}{l}\text { p value } \\
\text { OR }(95 \% \text { CI })\end{array}$ \\
\hline Subjects successfully treated & $72(96 \%)$ & $61(81 \%)$ & $0.010^{\star}$ \\
Subjects in whom treatment failed & $3(4 \%)$ & $14(19 \%)$ & $5.5(1.51$ to 20.06$)$ \\
\hline
\end{tabular}

${ }^{\star} \mathrm{p}<0.05$ eradication rate of group 1 versus group 2; OR: $5.5,95 \%$ CI: 1.51 to 20.06 .

$37^{\circ} \mathrm{C}$ under microaerophilic conditions, typical oxyidase and catalase positive colonies were identified by API Campy strips (bioMérieux), and subsequently tested for antibiotic sensitivity (E test). ${ }^{18}$ The following antibiotics were tested: ampicillin, tetracycline, metronidazole, and clarithromycin (AB Biodisk, Sweden). The minimum inhibitory concentration (MIC) interpretative values used to define resistance $(\mathrm{R})$ to each antibiotic are reported in table 2 .

Patients in group 1 were treated with ranitidine $(6 \mathrm{mg} / \mathrm{kg} /$ day, twice daily) if there was mild gastritis, or omeprazole $(1 \mathrm{mg} / \mathrm{kg}$ /day, once daily) if there was moderate or pronounced gastritis, or if there was an ulcer. Subjects with a positive culture also received two antibiotics chosen according to susceptibility at the standard dose for eight days (metronidazole $20 \mathrm{mg} / \mathrm{kg} /$ day; tetracycline $30 \mathrm{mg} / \mathrm{kg} /$ day if over 9 years of age; amoxycillin $50 \mathrm{mg} / \mathrm{kg} /$ day; clarithromycin $30 \mathrm{mg} / \mathrm{kg} /$ day). ${ }^{14}$

The group 1 subjects with a negative culture, and the patients in group 2 were treated with ranitidine or omeprazole according to the same criteria, and with amoxycillin (50 mg/kg/day) and clarithromycin $(30 \mathrm{mg} / \mathrm{kg} /$ day $)$ for eight days. ${ }^{14}$ The antisecretory treatment was continued for up to eight weeks in both groups, if symptoms persisted.
All patients were re-evaluated six months after finishing treatment, using the same investigation protocol as at diagnosis.

The main outcome of the study was evaluation of the efficacy of standard antibiotic treatment versus an antibiotic sensitivity assay based treatment.

STATISTICS

Statistical analysis was done using the $\chi^{2}$ test with Yates's correction. The odds ratio (OR) and the $95 \%$ confidence interval (CI) were calculated; $\mathrm{p}<0.05$ was considered significant.

\section{Results}

There was no significant difference between the ages of the two groups of subjects. The number of males and females was also similar in the two groups.

Table 1 details symptoms at presentation, endoscopic findings, histology, and Giemsa staining results of the sections for histology. In group 1, five subjects with negative Giemsa staining had positive cultures and urease tests. Seventy of 75 patients (93\%) had a positive urease test, but the five subjects with a negative test had a positive culture and positive histology. The 12/75 (16\%) patients with a negative culture had positive histology and urease test.

In the patients in whom endoscopic biopsy specimens were taken for the culture and antibiotic susceptibility testing, $H$ pylori grew in culture in 63/75 (84\%). Antibiotic resistance was detected in 53/63 isolates ( $84 \%$ ), with the highest percentage of resistance being observed towards metronidazole (56\%) (table 2).

Six months after treatment in 62/63 (98\%) subjects with a positive culture, all tests were negative (macroscopic appearance at endoscopy, urease test, histology, and culture).

Of the $12 / 75(16 \%)$ patients with an unsuccessful culture, 2/12 were lost at follow up. $H$ pylori was eradicated in the remaining 10 using the standard antibiotic combination of amoxycillin/clarithromycin. The infection was thus eradicated in $96 \%$ of the initial 75 subjects, in $99 \%$ of the 73 patients followed up, and in $98 \%$ of the subjects with a positive culture treated according to the antibiotic assays $(62 / 63)$ (table 3).

In all of the subjects in group 2, Giemsa staining of the biopsy specimens was positive for $H$ pylori. Eradication, verified six months later by means of endoscopy, urease test, and histology, was achieved in 61/75 subjects $(81 \%)$ (table 3). This was significantly lower than the percentage of eradication observed in group 1 ( $81 \%$ v $96 \%$, p < 0.05; OR: 5.50, CI: 1.51 to 20.06 ).

According to eradication these patients were divided into two subgroups, and compared in order to assess if there were any differences between the two subpopulations. Patient age, gender, and histological severity did not differ between those in whom eradication was or was not successful. The percentage of positive urease tests was significantly greater in those in whom eradication was successful $(57 \% v 92 \%$, $\mathrm{p}<0.05$; OR: 8.40, CI: 2.07 to 34.03; table 4 ). 
Table 4 Features at diagnosis of the two subgroups in group 2

\begin{tabular}{lll}
\hline & $\begin{array}{l}\text { Patients successfully treated } \\
(n=61)\end{array}$ & $\begin{array}{l}\text { Patients in whom treatment failed } \\
(n=14)\end{array}$ \\
\hline $\begin{array}{ll}\text { Chronological age (y) } \dagger \\
\text { Males/females }\end{array}$ & $9.29(7.60)$ & $13.10(7.60)$ \\
Positive urease tests & $38 / 23$ & $11 / 3$ \\
Histology & $56^{\star}$ & 8 \\
$\quad$ & 50 & 10 \\
$\quad$ Mild gastritis & 8 & 2 \\
Proderate gastritis & 3 & 2 \\
Duodenced gastritis & 2 & 0
\end{tabular}

${ }^{\star} \mathrm{p}<0.05$ versus the subjects who did not eradicate; OR: $8.40,95 \%$ CI: 2.07 to 34.03 . †Mean (SD).

In an analysis of the eradication rate in all patients for whom antibiotic susceptibility results were available compared with those treated blindly, the cure rate was significantly higher in the former group $(83 \%$ v 98\%, p < 0.05; OR: 12.58, CI: 1.60 to 98.45 ; table 3 ).

\section{Discussion}

The data reported in this study show that successful $H$ pylori eradication is obtained in a greater percentage of subjects, when an antibiotic susceptibility test is available and treatment is adjusted accordingly.

Antibiotic susceptibility was assessed using the $\mathrm{E}$ test, which is considered to be appropriate for testing $H$ pylori, and better than agar dilution and disc diffusion tests. ${ }^{18}$

Antibiotic resistance is a complex topic and has been discussed elsewhere. ${ }^{8}$ Dore and colleagues $^{19}$ reported unexpected antibiotic resistance in genotypically identical $H$ pylori isolates, based on the $\mathrm{E}$ test. ${ }^{19}$ There is uncertainty as to whether $H$ pylori should be eradicated given the existence of many mutants, and the fact that these are associated with clinically significant diseases in less than $20 \%$ of the population. ${ }^{20}$ Many would consider that children should be treated in order to prevent long term complications. ${ }^{12}$ Our policy is to treat children with $H$ pylori for eight days with combined therapy based on antibiotic susceptibility testing. We achieved eradication in $99 \%$ of cases. An additional advantage of susceptibility testing is that it may reduce the risk of creating $H$ pylori resistance. ${ }^{21}$

In the literature, to date, the percentage of antibiotic resistance varies between the continents, and within Europe. A significant proportion of our isolates were resistant to metronidazole, clarithromycin, ampicillin, or tetracycline, and a few were resistant to both clarithromycin and metronidazole, with the resistance to metronidazole being the most frequent. These ratios of resistance are higher than those published by most authors. Metronidazole resistance has been reported in up to $50 \%$ of isolates, ${ }^{379-11}$ clarithromycin resistance in $1-15 \%,{ }^{35-7} 9-1113$ amoxycillin resistance in $0.3-6 \%,{ }^{10}{ }^{11}{ }^{13}$ but resistance to tetracycline has not generally been reported. ${ }^{10}$ However, our results are comparable with those recently reported in adults with gastric ulcers. ${ }^{22}$

The success of treatment in $H$ pylori infection depends mainly on antibiotic sensitivity. With regard to the isolates tested in this study during the two time periods, sensitivity should have been similar as the patients were enrolled over a relatively short time interval and came from the same geographical area.

Treatment is generally more effective in children than in adults in whom the eradication rate has been reported at approximately $70-80 \%$ with triple therapy for two weeks. ${ }^{14}$ Combined treatment with amoxycillin and clarithromycin is considered a treatment of proven efficacy, ${ }^{14}$ and therefore represents a good comparison.

Among patients in whom antibiotic susceptibility testing was available, treatment failed in only one case. This patient also had Crohn's disease and was receiving long term steroid treatment.

We believe that treatment with amoxycillin is important, even with susceptibility testing, as it reduces the risk of antibiotic resistance as shown in subjects with persistent $H$ pylori infection previously treated with antibiotic regimens including clarithromycin. ${ }^{23}$

In this study, eradication was assessed six months after completion of treatment in order to reduce the possibility of $H$ pylori recurrence, although most authors consider assessment after four weeks, and some suggest 12 months. ${ }^{24}$

The only distinguishing feature of the patients in whom $H$ pylori was successfully eradicated in group 2 was the higher proportion of positive urease tests. This could reflect a better response to treatment in patients with a larger number of organisms. However, positive urease tests were found in both subgroups and this type of assay does not allow a quantitative analysis.

In conclusion, antibiotic susceptibility testing is worthwhile in childhood as a greater proportion of subjects are successfully cured. Testing may help to limit the development of antibiotic resistance and may avoid the need for repeated courses of treatment and reinvestigation. The extra cost of susceptibility testing is offset by the consequent financial savings. ${ }^{45} 25$

1 Forman D. Helicobacter pylori infection and gastric
carcinogenesis. Eur $\mathcal{f}$ Gastroenterol Hepatol 1992;4 (suppl):S31.

2 Blaser MJ, Chyou PH, Nomura A. Age at establishment of Helicobacter pylori infection and gastric carcinoma, gastric ulcer, and duodenal ulcer risk. Cancer Res 1995;55:562-5.

3 van Zwet AA, de Boer WA, Scheeberger PM, et al. Prevalence of primary Helicobacter pylori resistance to metronidazole and clarithromycin in the Netherlands. Eur f Clin Microbiol Infect Dis 1996;15:861-4.

4 Graham DY. Antibiotic resistance in Helicobacter pylori: implications for therapy. Gastroenterology 1998;115:1272-7.

5 Wreiber K, Olsson-Liljequist B, Engstrand L. Development of resistant Helicobacter pylori in Sweden. Tendency toward increasing resistance to clarithromycin. Lakartidningen 1999;96:582-4.

6 Nilsson F, Walder M. Antibiotic resistance of Helicobacter pylori in Malmo. Therapeutic success in spite of antibiotic resistance. Lakartidningen 1999;96:460-3.

7 Taylor DE, Jiang Q, Fedorak RN. Antibiotic susceptibilities of Helicobacter pylori strains isolated in the Provence of Alberta. Can F Gastroenterol 1998;12:295-8.

8 Lopez Vidal Y, Sigfrido Rangel Frausto M, Calva JJ. Antibiotic resistance of Helicobacter pylori in an infectious disease referral center. Rev Invest Clin 1998;50:19-24.

9 Megraud F. Antibiotic resistance in Helicobacter pylori infection. Br Med Bull 1998;54:207-16.

10 Jaup BH, Brandberg A, Stenquist B, et al. Antibiotic resistance among strains of Helicobacter pylori in Gothenburg. 95:279-81.

11 Adamek RJ, Suerbaum S, Pfaffenbach B, et al. Primary and acquired Helicobacter pylori resistance to clarithromycin, metronidazole, and amoxicillin-influence on treatment outcome. Am f Gastroenterol 1998;93:386-9. 
12 Van Zwett AA, Vandenbrouke-Grauls CMJ, Thijs JC, et al. Stable amoxicillin resistance in Helicobacter pylori. Lancet Stable amoxicillin

13 Teare L, Peters T. Antibiotic resistance in Helicobacter pylori. Lancet 1999;353:242.

14 Oderda G. Management of Helicobacter pylori infection in children. Gut 1998;43(suppl 1):S10-13

15 The report of the Digestive Health Initiative International Update Conference on Helicobacter pylori. Gastroenterology $1997 ; 113$ (suppl):S4-8

16 Current European concepts in the management of Helicobacter pylori infection. The Maastricht Consensus Report. Gut 1997;41:8-13.

17 Dixon MF, Genta RM, Yardley JH, Correa P, and the participants of the International Workshop on the histopathology of gastritis. Am F Surg Pathol 1996:20:1161-81.

18 Glupczynski Y, Labbé M, Hansen W, et al. Evaluation of the $\mathrm{E}$ test for quantitative antimicrobial susceptibility testing of Helicobacter pylori. 7 Clin Microbiol 1991;29:2072-5.

19 Dore MP, Osato MS, Kwon DH, et al. Demonstration of unexpected antibiotic resistance of genotypically identical Helicobacter pylori isolates. Clin Infect Dis 1998;27:84-9.
20 McCarthy DM. H. pylori infection in non-ulcer patients - to treat or not to treat. The case against patients - to treat or not to treat. The
treatment. Eur f Surg Suppl 1998;582:11-15.

21 Borody TJ, Shortis NP, Reyes E. Eradication therapies for Helicobacter pylori. f Gastroenterol 1998;33(suppl 10): 53-6.

22 Adamek RJ, Suerbam S, Pfaffenbach B, et al. Primary and acquired Helicobacter pylori resistance to clarithromycin, metronidazole, and amoxicillin-influence on treatment outcome. Am f Gastroenterol 1998;93:386-9.

23 Laine L, Suchower L, Frantz J, et al. Low rate of emergence of clarithromycin-resistant Helicobacter pylori with of clarithromycin-resistant Helicobacter pylori with
amoxycillin co-therapy. Aliment Pharmacol Ther 1998;12: 887-92.

24 Ishizuka J, Kato M, Sugiyama $T$, et al. The appropriate time for the assessment of Helicobacter pylori eradication. Nippon-Rinsho 1999;57:111-15.

25 Lam SK, Talley NJ. Helicobacter pylori consensus. Report of the 1997 Asia Pacific Consensus Conference on the management of Helicobacter pylori infection. 7 Gastroenterol Hepatol 1998;13:1-12.

\section{Rapid responses}

Letters on the following papers have been published recently as rapid responses on the $A D C$ website. To read these letters visit www.archdischild.com and click on "Read rapid responses":

Randomised controlled trial of infantile colic treated with chiropractic spinal manipulation. E Olafsdottir, S Forshei, G Fluge, et al. Arch Dis Child 2001;84:138-41.

Natural history of cardiovascular manifestations in Marfan syndrome. CDM van Karnebeek, MSJ Naeff, BJM Mulder, et al. Arch Dis Child 2001;84:129-37.

Violence against trainee paediatricians. JG Mackin, MR Ashton. Arch Dis Child 2001;84:106-8.

Scrotal temperature is increased in disposable plastic lined nappies. C-J Partsch, M Aukamp, W G Sippell. Arch Dis Child 2000;83:364-8.

If you would like to post an electronic response to these or any other articles published in the journal, please go to the website, access the article in which you are interested, and click on "eLetters: Submit a reponse to this article" in the box in the top right hand corner. 